REVIEW ARTICLE

P.M. White

J. Raymond

\title{
Endovascular Coiling of Cerebral Aneurysms Using "Bioactive" or Coated-Coil Technologies: A Systematic Review of the Literature
}

\begin{abstract}
SUMmARY: Second-generation coils have been available since 2002. We wanted to assess their performance and appraise available evidence. Therefore we performed a systematic review of the literature from 2002 to 2007 . There were 27 studies with a total of 2390 patients that met pre-specified inclusion criteria. All studies were classed as having a high risk of bias. There were no randomized trials and for most studies results were not independently assessed and follow-up periods were short (mean 7 months). There were large differences in demographic and aneurysm characteristics, making comparisons between coil cohorts difficult. Procedure-related morbidity and mortality were similar for all coil types. Hemorrhagic events during follow-up were few, in the range of $1 \% / y e a r$ for all coil types. The available literature is of poor quality and clinical series provide very little evidence in favor of second-generation coils. Positive randomized trial results are needed to justify routine clinical use. This systematic review illustrates the failure of the industry, the regulatory authorities, and the neurointerventional community combined to provide a reliable and prudent approach to the introduction of new devices.
\end{abstract}

S ince 2002, several coil manufacturers have introduced a variety of controlled detachment coil types specifically designed "to promote aneurysm healing" following cerebral aneurysm coiling or to improve the durability and angiographic results of coiling. Although they have been submitted to various regulatory agencies as "similar to a predicate device," once on the market, these coils were often claimed to be substantially different from or better than standard platinum coils. These devices include coils using polyglycolic (PGA) or combined polyglycolic/polylactic acid (PGLA), nylon/Dacron (DuPont, Wilmington, Del)/PGLA fibers, and hydrogel coating. None of these products were subjected to randomized controlled trial evaluation before being marketed extensively, ${ }^{1,2}$ and many were not even seriously tested in appropriate animal models. ${ }^{3}$ Many were sold at a substantial cost premium despite the lack of grade 1 evidence for equivalent safety, let alone of improved efficacy compared with the proved bare platinum coil technology. ${ }^{4,5}$ Several years later, randomized controlled trials are underway, but it will be some months before the first of these, the HydroCoil Endovascular Aneurysm Occlusion and Packing Study trial, ${ }^{6}$ reports follow-up results and approximately $1-6$ years before the others report. In an effort to clarify and critically appraise the existing published literature of case series, prospective single- or multicenter studies, and national or international registries, we have undertaken a systematic review. Such reviews reliably determine the quality and quantity of published work and can help direct/prioritize future research. ${ }^{7}$

\section{Received August 1, 2008; accepted August 8}

From the International Consortium of Neuroendovascular Centres [ICONE] (J.R., P.M.W.), Interventional Neuroradiology Research Unit, Department of Radiology, University of Montreal, CHUM Notre-Dame Hospital, Montreal, Canada; and Department of Clinical Neurosciences (P.M.W.), Western General Hospital, Edinburgh, UK.

Please address correspondence to P.M. White, MD, Consultant Neuroradiologist, Department of Neuroradiology, Western General Hospital, Edinburgh, EH4 2XU, UK; email: pmw@skull.dcn.ed.ac.uk

Indicates open access to non-subscribers at www.ajnr.org

DOI 10.3174/ajnr.A1324

\section{Materials and Methods}

\section{Search Strategy}

We performed a systematic review of the literature (paper/electronic) from 2002 to 2007 inclusive. A computerized search strategy of MEDLINE and EMBASE databases was undertaken; this was supplemented by hand searching of the 3 main journals in which most of the studies identified by the database search were published (American Journal of Neuroradiology, Stroke, and Neuroradiology) and reference lists of identified articles. Keywords and free text searched were the following: bioactive, Cerecyte, Matrix, HydroCoil, hydrogel, hydrogel-coated coil, PGA, polyglycolic acid, PGLA, polyglycolactic acid, fibered coil, Sapphire, and nexus in different combinations (by using the Boolean operator OR) in conjunction with (Boolean operator AND) the keywords "aneurysm" OR "coil," The search was then limited to adult humans and an English abstract. No additional reports were identified by hand searching of the journals. Searching of reference lists identified 1 additional study. A second author (J.R.) independently assessed the reproducibility of the search strategy.

\section{Eligibility}

The prespecified primary inclusion criteria that a reference had to meet were as follows: 1) the study evaluated coated-coil technology in cerebral aneurysm coiling, 2) a study of $\geq 10$ individuals; 3 ) a study published in a peer-reviewed journal, and 4) an original study (not a letter, review article, or editorial).

\section{Data Extraction}

All studies that met the primary criteria were then formally assessed, and data were extracted by 1 of the authors (P.M.W.) by using a standardized critical appraisal and data-extraction form (Fig 1). As a validation of the data-extraction process, a second author (J.R.) reviewed a random sample of $20 \%$ of the studies. The second author (J.R.) independently reviewed all the data extracted and assigned a second score to each study.

The data-extraction form was subdivided into 3 basic sections: 1 ) design and baseline population characteristics, 2) procedure, and 
Publication Reference:

Number patients in study Clinical status by WFNS Grade: $0=$

; 1 or $2=\quad ; 3=\quad ; 4$ or $5=$

$; 6=$ Sex breakdown Age breakdown

Number aneurysms in study Aneurysm profile: Ruptured [ \%] Unruptured [ \%]

Distribution of aneurysms described

Aneurysm sizes: Small $=\quad[\%]$; Medium $=$ $[\%] ;$ Large $=\quad[\%] ;$ Giant $=$ Inclusion \& Exclusion criteria adequately stated Study consent/IRB etc. described Unaccounted for patients?

\section{$\underline{\text { Procedural Details }}$}

Coated coil used: Proportion exclusively treated with CC: [ \% ] Packing Density (mean/median):

Assist device usage: Overall $=\quad[\%]$; Stent $=\quad[\quad \%]$; Balloon $=\quad[\%]$; Other $=\quad[\quad \%]$

Periprocedural complications: Overall Cx rate resulting in permanent deficit $=\quad[\%]$ Mortality rate $=\quad[\quad \%] \quad$ Combined $\mathrm{M} / \mathrm{M}$ rate $=[\quad \%]$ Perforation rate $=\quad[\%]$; T-Embolic event rate $=\quad[\%]$; Coil migration rate $=\quad[\%]$; Hydrocephalus rate $=\quad[\quad \%$;

Angio outcome: Montreal grade: $1=\quad[\%] ; 2=\quad[\%] ; 3=\quad[\%] ; 1 / 2=\quad[\quad \%]$

$\underline{\text { Results Data }}$

Independent assessment of angio outcome:

Independent assessment of clinical outcome:

Angiographic follow -up: \% with f'up =

Mean / Median follow-up (months) $=$

Rebleed rate $=\quad[\quad \%]$

Montreal grade: $1=\quad[\%] ; 2=\quad[\%] ; 3=\quad[\%] ; 1 / 2=\quad[\%]$

Clinical follow up: Scale used $=$

Proportion with dichotomised good outcome $=[\%]$

Aneurysm retreatment rate [up to 2 years]: actually reRx or advised/planned $=\quad[\%]$

QS Demographic data $\square$ QS Procedural data

[ 3 = Good; 2 = Adequate; 1 = Poor; $0=$ inadequate data to assess $]$

QS Results data $\square$ Overall Imp score (0-1)

QS $>4$ ?

Fig 1. Data extraction proforma.

3) outcomes. A score of $0-3(0=$ inadequate data to assess, $1=$ poor, $2=$ acceptable, $3=$ good) was assigned for each category (Fig 1$)$. This proforma method has been well described and enabled an objective and reproducible assessment to be made of each article. ${ }^{7-10}$ The quality scoring system was intrinsically weighted so that some items on the data-extraction form carried more weight than other items (Table 1). Weighting was used because some items relate more strongly to the reliability of results obtained than others-for instance, it is likely that authors will reliably report the coil studied (low weighting) but independent core lab assessment of angiographic outcomes is more reliable than operator self-assessment (high weighting). ${ }^{11}$ To score full (3) marks in a category, a study had to meet most of the highly weighted criteria and most of the lower weighted ones. To score 2 in a category, a study had to meet a majority of the high- and some lower weighted criteria and so on.

An additional score $(0,0.5$, or 1$)$ was assigned for "overall impression" based on how many of the following standards were judged to have been met: adequate reference list, objective discussion, discussion of limitations of the study, logical clear presentation of data, providing numerators/denominators as well as percentages, placing the study in context, and impact factor of the journal $>1.8$. The possible appraisal score was $0-10$, and a final percentage score was determined on the basis of the mean of the 2 quality scores awarded by the reviewers. A score of $>40 \%$ was required for inclusion. When the reviewers' disagreed on inclusion/exclusion, this disagreement was resolved by a consensus review of the article and the data-extraction forms. We did not set the bar for inclusion very high at all, and most studies following the usual scientific conventions of data description and presentation should have met these quality criteria.

\begin{tabular}{lc}
\hline $\begin{array}{l}\text { Table 1: Data extracted from studies meeting primary inclusion } \\
\text { criteria }\end{array}$ & Weighting (Low/High) \\
\hline Section/Subsection Category & \\
\hline Design, baseline data & $\mathrm{L}$ \\
No patients, aneurysms in study & $\mathrm{L}$ \\
Clinical status (WFNS) & $\mathrm{L}$ \\
Age/sex demography & $\mathrm{H}$ \\
Aneurysm location & $\mathrm{H}$ \\
Study inclusion/exclusion criteria & $\mathrm{L}$ \\
Consent/ethics described & $\mathrm{L}$ \\
Rupture/unruptured & \\
Size composition of aneurysms & $\mathrm{L}$ \\
Procedural data & $\mathrm{L}$ \\
Coils assessed & $\mathrm{L}$ \\
Exclusive coiling with coated coil & $\mathrm{H}$ \\
Packing density & $\mathrm{H}$ \\
Assist-device usage & $\mathrm{H}$ \\
Detail of procedural complications & \\
Procedural angio result & $\mathrm{H}$ \\
Outcome data & $\mathrm{H}$ \\
Independent angio assessment & $\mathrm{H}$ \\
Independent clinical assessment & $\mathrm{L}$ \\
Proportion with angio follow-up & $\mathrm{L}$ \\
Duration of angio follow-up & $\mathrm{H}$ \\
Detail on clinical tool, results & $\mathrm{L}$ \\
Angio results & $\mathrm{H}$ \\
Rebleed rate & Aneurysm retreatment rate \\
\hline Note:-H indicates high; L, lower weighting; WFNS, World Federation of Neurosurgeons.
\end{tabular}

\section{Data Analysis}

The following comparisons were made between the nonparametric data on coated coil types (where sufficient data could be extracted) by 


\begin{tabular}{lcc}
\hline \multicolumn{2}{c}{ Table 2: Results of computerized search strategy* } \\
\hline Search Term & $\begin{array}{c}\text { MEDLINE } \\
\text { References }\end{array}$ & $\begin{array}{c}\text { EMBASE } \\
\text { References }\end{array}$ \\
\hline Bioactive & 5 & 5 \\
Cerecyte & 6 & 5 \\
Fibered & 2 & 11 \\
GDC fibered & 0 & 0 \\
Hydrogel & 12 & 19 \\
HydroCoil & 21 & 21 \\
Matrix & 47 & 53 \\
Nexus & 0 & 0 \\
Nylon & 1 & 2 \\
PGLA or PGA & 7 & 7 \\
Sapphire & 2 & 2 \\
\hline
\end{tabular}

Note:-GDC indicates Guglielmi detachable coil; PGLA, polyglycolic/polylactic acid; PGA, polyglycolic acid

* NB search terms were combined with "aneurysm" OR "coil" and limited to the years 2002-2007 inclusive in human subjects and English-language publications.

using the $\chi^{2}$ test or, when appropriate, the Fisher exact test: demographic and aneurysm characteristics, procedural complication rates resulting in permanent morbidity/mortality (combined rate), clinical outcomes, angiographic incomplete occlusion rates on follow-up, aneurysm rebleeds, and retreatment rates. Due to the marked heterogeneity and uncontrolled nature of the data, a $P$ value of $<.01$ was prespecified to indicate a statistically significant difference (rather than the more commonly quoted $P<.05$ ).

\section{Results}

Results of the computerized search of EMBASE and MEDLINE are given in Table 2. Two hundred twenty-eight references for "coated" coil used in cerebral aneurysm treatment were identified by the search strategy. Removing duplicates and irrelevant references, such as letters or editorials or other reports that did not meet the primary inclusion criteria, left 32 studies to be critically reviewed. The sensitivity of the electronic search strategy was $97 \%$ (it identified 31/32 studies).

Of these studies, 15 were on Matrix (PGLA-coated coils from Boston Scientific, Natick, Mass), ${ }^{12-26} 11$ were on hydrogel-coated coils (HydroCoil embolic system; MicroVention, Aliso Viejo, Calif), ${ }^{27-37} 1$ was on both hydrogel and Matrix, ${ }^{38} 2$ were on Sapphire fibered coils (ev3, Irvine, Calif), ${ }^{39,40}$ and 3 were on Cerecyte (PGA-coated coils; Micrus, Sunnyvale, Calif). ${ }^{41-43}$ The study on PGLA- and hydrogel-coated coils was excluded because it was impossible to separate information on the 2 coil types from bare platinum data, let alone from each other; thus in terms of results by coated coil, it was impossible to assess. One study on PGLA scored 30\% and was excluded, 1 short report on PGA-coated coils scored 30\% and was excluded, and 1 early technical report on hydrogel-coated coils scored $20 \%$ and was excluded. One fibered coil article was a similar series from the same unit, so the report focused on fibered coils was the 1 formally reviewed. In just 1 case (3\%), the reviewers disagreed on inclusion/exclusion, and this disagreement was resolved by a consensus review with an agreement not to include it. The other 26 studies were included. Median quality assessment scores for included studies were $60 \%$ (range, $45 \%-90 \%$ ) with no difference in median scores between studies on different coil types.

\section{PGLA-Coated Coils (Matrix)}

Fourteen studies on 1119 patients and 1215 aneurysms treated by using PGLA-coated coils were included. (See Table 3 for a summary of the major aneurysm demographics and procedural safety results and Table 4 for angiographic data.) A detailed breakdown by clinical grade was provided in 8 studies. Among the 393 patients with subarachnoid hemorrhage (SAH) in these 8 studies, $245(62 \%)$ had good grades (World Federation of Neurology [WFNS] or Hunt and Hess [HH] scale grade 1 or 2). Size categorization of aneurysms was quite variable among studies. However, in 9 studies, it was possible to categorize aneurysms into the following 3 size groups: $<10$ $\mathrm{mm}, 10-25 \mathrm{~mm}$, and $>25 \mathrm{~mm}$. There were insufficient data to categorize aneurysms regarding neck size. Only 4 studies assessed packing density, and the median packing density was $32 \%$ (range, 29\%-40\%). Nine studies reported stent-device usage, and 9 studies reported balloon-remodelling use (data derived from 10 studies in all)_-Table 3.

All 14 studies reported complications to some extent, though data were quite limited in 2 of them. No studies reported hydrocephalus. For the purposes of the systematic review, periprocedural coil migration events are included with thromboembolic complications. Many of these did not result in a clinical event, or even if they did, the events were transient. Therefore, procedural complications resulting in a permanent deficit are given as a separate category in Table 3.

Eight studies reported rebleeding-Table 5. There were 7 rebleeds from the target aneurysm (coiled with PGLA-coated coils), all occurring within 1 year of the procedure. Unfortunately, it was not possible to calculate this by ruptured/unruptured status because there were insufficient data presented separately on these groups in the studies. Eight studies reported aneurysm retreatment following coiling-Table 5. The retreatment group did include some giant aneurysms, which are highly likely to recur following endovascular treatment. The precise number of retreatments of giant aneurysms could not be extracted and quantified from the published data.

Eight studies formally reported clinical outcomes by using a recognized outcome scale (Modified Rankin Scale [mRS] in 3, Glasgow Outcome Scale [GOS] in 4, and both in 1).

\section{Hydrogel-Coated Coils (HydroCoil)}

Ten studies on 687 patients and 720 aneurysms were included. (See Table 3 for a summary of the major aneurysm demographics and procedural safety results and Table 4 for angiographic data.) A breakdown by clinical grade was provided in only 5 studies. Among the 211 patients with SAH in these 5 studies, $116(55 \%)$ had good grades (WFNS or $\mathrm{HH}$ scale grades 1 or 2). Again, size categorization of aneurysms was quite variable among studies. However, in 6 studies, it was possible to categorize aneurysms into the following 3 size groups: $<10 \mathrm{~mm}, 10-25 \mathrm{~mm}$, and $>25 \mathrm{~mm}$. There were insufficient data to categorize aneurysms regarding neck size. Seven studies assessed packing density, and the median packing density was 68\% (range 50-85\%). Seven studies (not the same 7), including 576 aneurysms, reported assist-device usage-Table 3. All 10 studies reported complications (Table 3). Four studies reported hydrocephalus, though the degree of detail provided varied. In these 4 studies, there were overall 10 cases of hydrocephalus reported in 285 patients (3.5\%), and 


\begin{tabular}{|c|c|c|c|c|}
\hline & PGLA Coated & Hydrogel Coated & PGA Coated & Fibered \\
\hline & Num/Denom (\%) & Num/Denom (\%) & Num/Denom (\%) & Num/Denom (\%) \\
\hline Ruptured aneurysms & $728 / 1215(60)$ & $353 / 720$ (49) & $98 / 123(80)$ & DNE \\
\hline Aneurysm size & Given in $11 / 14$ studies & Given in $6 / 10$ studies & Given in $1 / 2$ studies & 474 aneurysms \\
\hline Small/medium (<10 mm) & 745/899 (82.9) & 233/343 (68) & $67 / 68(98.5)$ & $99<5 \mathrm{~mm}(23.5)$ \\
\hline Large $(10-25 \mathrm{~mm})$ & 149/899 (16.6) & $92 / 343(27)$ & $1 / 68(1.5)$ & $316=5-25 \mathrm{~mm}(75.1)$ \\
\hline Giant (>25 mm) & $5 / 899(0.6)$ & $18 / 343(5)$ & $0 / 123(0)$ & $6(1.4)$ \\
\hline Overall assist device use & 215/957 (22) & $132 / 576(23)$ & DNE & DNE \\
\hline Stent & $113 / 942(12)$ & $50 / 132(9)$ & $5 / 123(4)$ & NS \\
\hline Balloon & $102 / 957(11)$ & $81 / 132(14)$ & NS & NS \\
\hline Other & 0/957 (0) & $1(0.2)$ & NS & NS \\
\hline Procedure-related morbidity, mortality rate & $53 / 1199(4.7)$ & 20/687 (2.9) & $3 / 67(4.5)$ & $25 / 463(5.4)$ \\
\hline Permanent procedural morbidity & 41/1199 (3.4) & $18 / 687(2.6)$ & $0 / 67(0)$ & $18 / 463(3.9)$ \\
\hline Procedure-related mortality & $12 / 1199(1.1)$ & $2 / 687(0.3)$ & $3 / 67(4.5)$ & $7 / 463(1.5)$ \\
\hline Aneurysm perforation & $29 / 1215(2.5)$ & $9 / 720(1.3)$ & $3 / 67(4.5)$ & NS \\
\hline Thromboembolic event & $129 / 1215(10.6)$ & $50 / 720(6.9)$ & $12 / 123(9.8)$ & $38 / 474(8)$ \\
\hline Hydrocephalus & NS & $10 / 285(3.5)$ & NS & NS \\
\hline
\end{tabular}

Note:-Num/Denom indicates numerator/denominator; NS, not stated; DNE, data not extractable.

\begin{tabular}{|c|c|c|c|c|}
\hline & PGLA Coated & Hydrogel Coated & PGA Coated & Fibered \\
\hline Procedural Angio Result & Num/Denom (\%) & Num/Denom (\%) & Num/Denom (\%) & Num/Denom (\%) \\
\hline Montreal grade 1 or 2 & $946 / 1178$ (80) & $614 / 716(86)$ & $120 / 123(98)$ & $452 / 476$ (95) \\
\hline Montreal grade 3 & 232/1178 (20) & $102 / 716(14)$ & $3 / 123(2)$ & $22 / 476(5)$ \\
\hline Proportion with angio control & $668 / 1215(55)$ & $490 / 720(71)$ & $101 / 123(82)$ & $156 / 474(33)$ \\
\hline Mean duration of follow-up & 7.6 (months) & 7.5 (months) & 6 (months) & 6 (months) \\
\hline \multicolumn{5}{|l|}{ Control angio result } \\
\hline Montreal grade 1 or 2 & $503 / 668(75)$ & $407 / 490(83)$ & $91 / 101(90)$ & (71) grade 1 \\
\hline Montreal grade 3 & $165 / 668(25)$ & $83 / 490(17)$ & $10 / 101(10)$ & (29) grade $2 / 3$ \\
\hline \multicolumn{5}{|l|}{ Table 5: Outcomes } \\
\hline & PGLA Coated & Hydrogel Coated & PGA Coated & Fibered \\
\hline & Num/Denom (\%) & Num/Denom (\%) & Num/Denom (\%) & Num/Denom (\%) \\
\hline Clinical outcome reported & 8/14 studies & $2 / 10$ studies & 0/2 studies & $1 / 1$ \\
\hline Good outcome rate & $307 / 495(62)$ & $58 / 63(92)$ & NS & $353 / 463(76)$ \\
\hline Rebleeds & 7 in 8 studies & 3 in 9 studies & 0 in 2 studies & NS \\
\hline Rebleed rate (\% pa) & 7 in 432 years of follow-up (1.6) & 3 in 256 years of follow-up (1.2) & 0 in 51 years of follow-up (0) & NS \\
\hline Aneurysm retreatment & 63 in 8 studies & 13 in 7 studies & 1 in 1 study & DNE \\
\hline By total aneurysms & $63 / 634(10)$ & $13 / 400(3)$ & $1 / 55(2)$ & \\
\hline By aneurysms followed up & $63 / 472(13)$ & $13 / 268(5)$ & $1 / 55(2)$ & \\
\hline
\end{tabular}

Note:- pa indicates per annum.

overall just over half (145/285) of the patients presented with a ruptured aneurysm. However, it was not possible to extract a breakdown of hydrocephalus by rupture status in most of these series. Nine studies reported rebleeding-Table 5. There were 3 cases, all occurring within 1 year of the procedure. Unfortunately it was not possible to calculate this by ruptured/ unruptured status because there were insufficient data presented separately on these groups in the studies. Nine studies reported aneurysm retreatment following coiling-Table 5 . Although this retreatment group did include some giant aneurysms, as for PGLA coils, the precise figure could not be quantified from the published data. Only 2 studies formally reported clinical outcomes by using a recognized outcome scale ( $\mathrm{mRS}$ in both).

\section{PGA-Coated Coils (Cerecyte)}

Two studies on 121 patients with 123 aneurysms treated by using PGA-coated coils were included. (See Table 3 for a summary of the major aneurysm demographics and procedural safety results and Table 4 for angiographic data.) Among the 98 patients with SAH in these 2 studies, 71 $(72 \%)$ were in grades (WFNS or HH scale grade 1 or 2 ). Neither study reported packing density. Both studies mentioned stent usage but gave no data on other assist devices. Only 1 of the 2 studies gave a more detailed breakdown on procedural complications, though both presented data on thromboembolic complications (Table 3). No rebleeds were reported up to 6 months. Only 1 study reported the retreatment rate following coiling, and this was low at 1 case $(1.8 \%)$ by 6 months. Neither study reported clinical outcomes using a recognized outcome scale.

\section{Fibered-Controlled Detachment Coils}

Only 1 of the fibered-controlled detachment coil studies was included. This included 463 patients with 474 aneurysms treated by fibered-controlled detachment coils. No articles, 
other than an earlier report of the series of this center, were identified that met the systematic review primary inclusion criteria (cutoff date the end of 2007). Data on fibered and bare platinum coils were frequently combined in this article, so it was not possible to extract data on the proportion of patients with ruptured aneurysms treated with fibered coils or on the clinical grade. The aneurysm demographic/procedure data that could be extracted for the cohort of 463 patients treated with fibered coils are presented in Table 3. Angiographic data are presented in Table 4, and clinical outcome data (GOS), in Table 5. With regard to angiographic follow-up, a striking finding in this study was that the rate of follow-up was so different for aneurysms treated with bare platinum and fibered coils at $60 \%$ and $33 \%$, respectively. No explanation or comment on this marked discrepancy was given. Also the Montreal grade ${ }^{44} 1,2$, and 3 results were presented separately for immediate procedural results, but then grades 2 and 3 were combined for follow-up, so it was not possible to present follow-up angiographic data as for the other coated coil typesdichotomized into complete/near-complete occlusion versus incomplete. No extractable data were presented on rebleeding or on aneurysm retreatment.

\section{Comparisons between Coated-Coil Cohorts}

Demographic and Aneurysm Characteristics. There were extreme differences in the proportion of ruptured/unruptured aneurysms as well as in the sizes of treated aneurysms among the amalgamated series of studies of various coils $(P<.001)$.

Procedure-Related Combined Morbidity/Mortality Rates. These morbidity/mortality rates varied from $2.9 \%$ to $5.4 \%$, but none of the differences were statistically significant by the prespecified definition for the systematic review $(P<$ $.01)$.

Clinical Outcomes. There was a significant difference among hydrogel-coated coils (92\% good outcome) and PGLA (62\% good outcome, $P=.0003$ ) and fibered coils (76\% good outcome, $P=.004)$. The difference between fibered coils and PGLA was also statistically significant $(P<.01)$. However, only a small number of studies reported outcomes, especially for hydrogel-coated coils.

Immediate Angiographic Results. There were large differences in the immediate angiographic results reported between PGA-coated coils and hydrogel-coated coils, as well as between PGA-coated and PGLA-coils $(P<.001)$.

Angiographic Follow-Up Outcomes. There was no significant difference in the proportion of aneurysms incompletely coiled on angiographic control between hydrogel-coated coils $(17 \%)$ and PGA-coated coils $(10 \%)(P=.1)$. There was a significant difference in incomplete rates between hydrogel $(17 \%)$ and PGLA $(25 \%)(P=.001)$ and also between PGAcoated $(10 \%)$ and PGLA-coated coils $(25 \%)(P=.001)$.

Rebleeding Rates. Data were just too limited for PGA and fibered coils to make any comparisons of rebleeding rates. There was no significant difference between PGLA- (7 in 665 patients reported) and hydrogel-coated coils ( 3 in 503 patients reported) $(P=.4)$.

Retreatment Rates. There was a significant difference between retreatment rates in hydrogel-coated (5\%) and PGLAcoated coils $(13 \%)(P=.0003)$ but not between hydrogelcoated $(5 \%)$ and PGA-coated coils $(2 \%)(P=.3)$; the significance of the difference between PGA- and PGLA-coated coils was borderline $(P=.01)$.

\section{Discussion}

First, we need to issue a strong "health warning": These data were extracted from uncontrolled mostly self-assessed case series and are, at best, exploratory. Far from guiding clinical actions, they can be used only to provide hypotheses for future trials. There were extreme differences in patient and aneurysm characteristics, in study methodologies (generally poor), and quantities of data between studies and cohorts, so these results must be viewed with considerable caution. In particular, the amalgamated coil series were vastly different for many risk factors that are believed to have impact on procedural and periprocedural morbidity and mortality and on initial and follow-up angiographic results, including the proportion of ruptured/unruptured cases, the number of aneurysms in various size categories, and the proportion of patients followed up. ${ }^{44}$ Very incomplete data were available on assist-device usage. Furthermore, there was considerable inconsistency in the data reported (demonstrated by the varying numbers of studies reporting the different outcomes assessed and thus the varying denominator values in Tables 3-5). One particularly disappointing feature was the overall low level of clinical and angiographic follow-up reported. It is clearly unacceptable that the overall angiographic follow-up rate reported on these new unproven technologies was so low at $56 \%$ and the duration of follow-up, so short at 7.3 months.

None of the data published on coated coils to the end of 2007 were from randomized controlled trials. This in itself is an indictment of the state of scientific evidence in the neurointerventional field, despite the good example set early on in the coiling experience by the International Subarachnoid Aneurysm Trial (ISAT), ${ }^{4,5}$ albeit things are now improving. The absence of control groups and the lack of randomization are not the sole deficiencies identified. By the Cochrane review methodology, all studies reviewed were classified as level C with a high risk of bias. ${ }^{9}$ Only 3 studies (9\%) had any independent assessment of outcomes (2 studies on hydrogel-coated coils and 1 on PGLA coils had an independent core lab). Only $6 / 26$ studies had a quality score above $60 \%$, and 9/26 had a score of $\leq 50 \%$. Only $1 / 26$ studies had a domain score of 3 (good) in $>1$ data category, despite a very generous review/ scoring policy.

Despite the avowed aims of assessing "safety and efficacy," none of the series attempted to prespecify 1 or more research questions that would 1) allow an estimate of the sample size necessary to answer these questions, 2) control the error that would come with the data, and 3) set a limit to the claims of the conclusions. Claims of efficacy and safety are unsubstantiated and based on series too small to conclude anything with any confidence. More disturbing, in the light of current policies regarding clinical research involving human subjects, is the fact that though most authors would acknowledge the lack of evidence regarding the safety and efficacy of the new material in the introduction of their report, they would still proceed with an uncontrolled study (which could be called an "experiment") on their patients. In most cases, this was done without any statement regarding a submission to ethics review and without, presumably therefore, obtaining a specific research- 


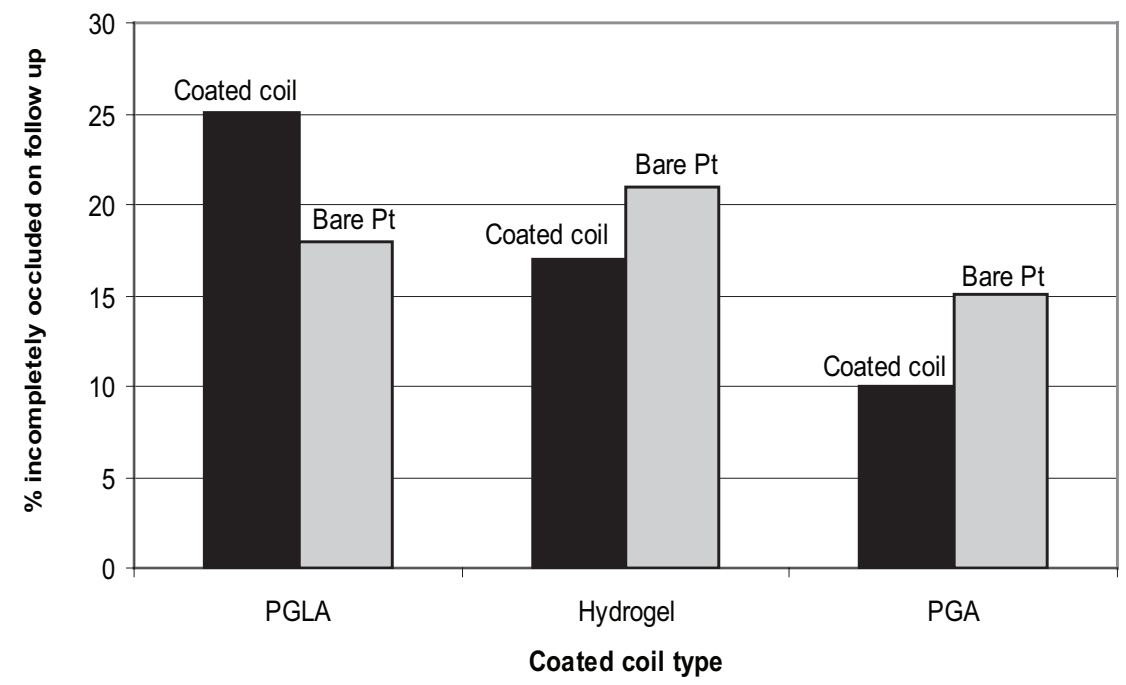

Fig 2. Incomplete occlusion rate observed with coated coils by comparison with the incomplete occlusion rate expected for a "virtual series" of bare platinum coils in a size-matched aneurysm cohort.

related informed consent from participants, which would acknowledge that the authors were trying a new material with unknown risks. This may perhaps be explained by confusion between using devices that have passed arbitrary (and often deficient) regulatory requirements and what it is ethical to do in clinical practice and research. The systematic review only represents a very small proportion of patients who have been submitted to the new materials over a 5-year period, without any clear evidence of benefit. This leads to a feeling of disquiet, to say the least, once the reader realizes that dozens of institutions and physicians were simultaneously performing poorly planned "experiments," without any possibility of providing a convincing answer to important clinical questions and mostly without consulting and collaborating with each other. One must come to the conclusion that neurointerventionists, as a group of clinicians respectful of their patients, must find a way to do things better. ${ }^{1-3,45}$

So if it is a case of generally poor data in, is it necessarily poor data out and thus a waste of time doing a systematic review? Is it possible to turn the flock of ugly ducklings into swans?

Because the structured quality assessment process, by using a specially designed data-extraction form, results in an objective measure of study quality, the method itself provides a useful analysis of existing literature. ${ }^{46}$ There are moderately large numbers of patients/aneurysms to draw on (2390/2532, respectively) from nearly 30 peer-reviewed studies. Despite many limitations, a systematic review methodology may allow a useful overview of new-device use and some comparisons to be made, though one must be disciplined to refrain from hasty conclusions. So, if anything can be said about this literature, how do the coated-coil technologies compare? Due to the limitations of the raw data, we are here condemned to proceed with questionable comparisons and extrapolations, attempting to adjust for various risk factors purported to have an impact on complications and recurrences.

Overall procedural morbidity/mortality rates are comparable at $2.9 \%-5.4 \%$ and are reassuringly in line with those from the bare platinum literature and early randomized controlled trial evidence for coated coils. ${ }^{4,6,44,47-50}$ The fluctuations between coated-coil types in perforation and thromboembolic complication rates are modest and likely to represent statistical noise (because numbers are rather small), the presence of heterogeneity, or bias. One must remember that very large controlled trials are necessary to exclude a clinically significant difference in events with such a low frequency. Hydrocephalus was reported for hydrogel-coated coils in only $4 / 10$ studies (at a $3.5 \%$ rate overall) but was just not mentioned in any studies on the other coated coil types. Therefore, the systematic review can add no useful information on the relationship between coated coils and hydrocephalus rates. Given the discrepancies between case series and the fact that divergences for various risk factors can pull in different directions, trying to adjust for heterogeneity becomes a highly speculative endeavor. We have attempted to provide comparisons between coils by using "virtual control" platinum groups adjusted for only 1 factor, the size categories. Most studies of angiographic remnants/ recurrences in bare platinum coils indicate rates of $15 \%-19 \%$ by 6 months and approximately $20 \%$ by 18 months with far higher rates in large than in small aneurysms. ${ }^{44,47-54}$

Thus Fig 2 is a comparison between the observed results for coated-coil cohorts and the expected results for bare platinum coils for similar aneurysm-size cohorts-the "virtual control" platinum groups, with the assumption made for bare platinum coils of a $15 \%$ recurrence rate for small/medium aneurysms, a 30\% recurrence rate for large aneurysms, and a 50\% rate for giant aneurysms. Again one must remember that this is not hard evidence, but extrapolation. An increased prevalence of large/giant aneurysms would be expected to result in higher recurrence and retreatment rates because it has been demonstrated that lesions prone to recurrence such as these have a $34 \%-50 \%$ recurrence rate. ${ }^{44}$ Conversely, series of smaller aneurysms were also prone to include a larger proportion of ruptured aneurysms, another risk factor for recurrences. ${ }^{47,48}$ Despite the very different aneurysm size profiles, the angiographic follow-up results for hydrogel- and PGAcoated coils compared with bare platinum are similar (Fig 2). In comparison, PGLA coils, with an in-between aneurysm size 
profile, had significantly lower complete/near-complete occlusion rates. However, the marked disparities between the aneurysm cohorts may account for most of the differences despite the calculated $P$ values; the cohorts are particularly discrepant between PGA-coated coils and the others.

The retreatment rate is a relatively hard end point. An aneurysm either has been retreated or it has not. However, retreatment policies do vary substantially between units. ${ }^{55,56}$ Due to the heterogeneous mix of aneurysms and the wider use of assist devices in the systematic review, it is not possible to draw any meaningful comparisons with the ISAT data on retreatment rates (where retreatment in the endovascular arm was $11 \%$ at 1 year but in a very different aneurysm population). ${ }^{4,555}$ The retreatment rate in aneurysms followed up was $13 \%$ for PGLA, $5 \%$ for hydrogel, and 2\% for PGA. The problem of not comparing like with like in uncontrolled series means that the significantly lower retreatment rate for hydrogel- and PGA-coated coils compared with PGLA-coated coils needs to be viewed with skepticism. On the flawed evidence to date, overall the retreatment rate with coated coils seems to be little/no improvement over bare platinum despite advances in coil technologies (complex shapes, coils of varying softness) and access/assist devices all aimed at reducing recurrence and retreatment rates. Explanations for this might include the facts that early PGLA studies were on the Matrix Version 1, which was undoubtedly more difficult to use and pack with than the revised Version 2, and that early hydrogel studies used the much more limited range of hydrogel-coated coils then available, which were stiffer and harder to obtain gel-neck coverage - a factor linked to outcome in 1 independently assessed registry study. ${ }^{27}$

Reassuringly, reported rebleed rates in the patients with SAH were low, which is in line with the bare platinum coiling literature, ${ }^{2,55,56}$ albeit follow-up in the coated-coil data is limited to the first year or less. There was no appreciable difference among coil types for which data were available, but only 19/26 studies documented rebleeds.

\section{Limitations of the Current Study}

One limitation is that only articles published in peer-reviewed journals were included in the systematic review, thus introducing publication bias. Our original intention was to include unpublished literature by approaching authors of abstracts on coated coils presented at major international neuroradiology congresses for additional information and original data. In contrast to the registration process for randomized trials (so one can accurately search for those not published), there is no register of registries let alone of prospective and retrospective case studies, so we could not assess at all the validity or precision of any search for unpublished data. Therefore, it was extremely uncertain how representative of unpublished data this strategy would be.

A second limitation is that only the English language was searched. However, many reports of new technologies in nonEnglish language journals eventually appear in English language ones. ${ }^{8,10}$ Also until late 2006, many coated coils were either not available or not widely used in major countries, including Japan, China, and India. Many non-English European journals publish abstracts or dual publish in English, and thus, the article could be picked up by the search strategy used.

\section{Conclusions}

The published literature to the end of 2007 provides no highquality (grade A or B) evidence for the safety or efficacy of bioactive/coated coils. Conversely, there is little indication that coated coil technologies are associated with increased procedural complications but also little evidence for any appreciable improvement in angiographic outcomes. This solid randomized controlled trial evidence with independently assessed outcomes is urgently needed. Until available, it is the authors' opinion that these coils should be used sparingly, if at all, outside an ethically and scientifically approved randomized trial (or in exceptional circumstances in other ethically approved studies with independent outcome assessment and informed patient consent). This review clearly demonstrates the past failure of the device industry, the regulatory authorities, and the neurointerventional community combined, to provide a prudent approach to the introduction of new technologies and to obtain reliable knowledge on new devices, and it provides a good illustration of the hype effect. ${ }^{57}$ As a community of physicians and allied professionals, we must find ways to coordinate our efforts to progress in a more scientific and ethical manner for the ultimate benefit of our patients.

\section{References}

1. Cloft HJ. Have you been smoking something that is biologically active? AJNR Am J Neuroradiol 2006;27:240-42

2. Raymond J, Guilbert F, Weill A, et al. Safety, science, and sales: a request for valid clinical trials to assess new devices for endovascular treatment of intracranial aneurysms. AJNR Am J Neuroradiol 2004;25:1128-30

3. Raymond J, Salazkin I, Gevry G, et al. Interventional neuroradiology: the role of experimental models in scientific progress. AJNR Am J Neuroradiol 2007;28:401-05

4. Molyneux A, Kerr R, Sandercodk P, et al, for the ISAT Collaborative Group International Subarachnoid Aneurysm Trial (ISAT) of neurosurgical clipping versus endovascular coiling in 2143 patients with ruptured aneurysms: a randomised trial. Lancet 2002;360:1267-74

5. Molyneux A, Kerr RS, Yu LM, et al, for the ISAT Collaborative Group. International Subarachnoid Aneurysm Trial (ISAT) of neurosurgical clipping versus endovascular coiling in 2143 patients with ruptured intracranial aneurysms: a randomised comparison of effects on survival, dependency, seizures, rebleeding, subgroups, and aneurysm occlusion. Lancet 2005;366:809-17

6. White PM, Lewis SC, Nahser H, et al. HydroCoil Endovascular Aneurysm Occlusion and Packing Study (HELPS trial): procedural safety and operatorassessed efficacy results. AJNR Am J Neuroradiol 2008;29:217-23. Epub 2008 Jan 9

7. Irwig L, Tosteson A, Gatsonis C, et al. Guidelines for meta analysis evaluating diagnostic tests. Annals Intern Med 1994;120:667-76

8. Kelly S, Berry E, Roderick P, et al. Use of Bias in Assessing Study Validity for Systematic Reviews of Diagnostic Performance Studies of Medical Imaging: Proceedings of the 15th Annual Meeting of the International Society of Technology Assessment in Health Care, Edinburgh, UK, 20-23 June 1999. Macclesfield, UK: Gardiner-Caldwell Communications; 1999:93

9. Higgins JPT, Green S, eds. Cochrane Handbook for Systematic Reviews Version 5.0. (updated February 2008). The Cochrane Collaboration 2008. Available at: www.cochrane-handbook.org. Accessed May 10, 2008

10. White PM, Wardlaw JM, Easton V. Can non-invasive imaging accurately depict intracranial aneurysms? A systematic review. Radiology 2000;217:361-70

11. Rothwell PM, Slattery J, Warlow CP. A systematic review of the risks of stroke and death due to endarterectomy for symptomatic carotid stenosis. Stroke $1996 ; 27: 260-65$

12. Lubicz B, Leclerec X, Gauurit JY, et al. Endovascular treatment of intracranial aneurysms with Matrix coils: a preliminary study of immediate post-treatment results. AJNR Am J Neuroradiol 2005;26:373-75

13. Taschner CA, Leclerc X, Rachdi $H$, et al. Matrix detachable coils for the endovascular treatment of intracranial aneurysms: analysis of early angiographic and clinical outcomes. Stroke 2005;36:2176-80

14. Murayama Y, Vinuela F, Ishi A, et al. Initial clinical experience with Matrix detachable coils for the treatment of intracranial aneurysms. J Neurosurg 2006;105:192-99

15. Niimi Y, Song J, Madrdi M, et al. Endosaccular treatment of intracranial aneurysms using Matrix coils: early experience and mid term follow-up. Stroke 2006;37:1028-32 
16. Rivet DJ, Moran CJ, Mazumdar A, et al. Single-institution experience with Matrix coils in the treatment of intracranial aneurysms: comparison with same-center outcomes with the use of platinum coils. AJNR Am J Neuroradiol 2007;28:1736-42. Epub 2007 Sep 20

17. Kimchi TJ, Willinsky RA, Spears J, et al. Endovascular treatment of intracranial aneurysms with Matrix coils: immediate posttreatment results, clinical outcome and follow-up. Neuroradiology 2007;49:223-29

18. Mitra D, Herwardkar A, Soh C, et al. Follow-up of intracranial aneurysms treated with Matrix detachable coils: a single-center experience. AJNR Am J Neuroradiol 2007;28:362-67

19. Linfante I, Akkawi NM, Perlow A, et al. Polyglycolide/polylactide-coated platinum coils for patients with ruptured and unruptured cerebral aneurysms: a single-center experience. Stroke 2005;36:1948-53. Epub 2005 Jul 28

20. Wong GKC, Yu SCH, Poon WS. Clinical and angiographic outcome of intracranial aneurysms treated with Matrix detachable coils in Chinese patients. Surg Neurol 2007;67:122-26

21. Deng J, Zhao Z, Gao G. Periprocedural complications associated with endovascular embolisation of intracranial ruptured aneurysms with Matrix coils. Singapore Med J 2007;48:429-33

22. Katsaridis V, Papagiannaki C, Violaris C. Guglielmi detachable coils versus Matrix coils: a comparison of the immediate posttreatment results of the embolization of 364 cerebral aneurysms in 307 patients-a single-center, singlesurgeon experience. AJNR Am J Neuroradiol 2006;27:1841-48

23. Rossitti S. Endovascular coiling of intracranial aneurysms using bioactive coils: a single-center study. Acta Radiol 2007;45:565-70

24. Pierot L, Bonafe A, Bracard S, et al. Endovascular treatment of intracranial aneurysms with Matrix detachable coils: immediate posttreatment results from a prospective multicenter registry. AJNR Am J Neuroradiol 2006;27: 1693-99

25. Kang HS, Han MH, Kwon BJ, et al. Short-term outcome of intracranial aneurysm treatment with polyglycolic acid/lactide copolymer-coated coils compared to historical controls treated with bare platinum coils: a single-center experience. AJNR Am J Neuroradiol 2005;26:1921-28

26. Fiorella D, Albuquerque FC, McDougall CG. Durability of aneurysm embolization with Matrix detachable coils. Neurosurgery 2006;58:51-58

27. Cloft $\mathrm{H}$, for the HEAL Investigators. HydroCoil for Endovascular Aneurysm Occlusion (HEAL) study: 3-6 month angiographic follow-up results. AJNR Am J Neuroradiol 2007;28:152-54

28. Geyik S, Yauuz K, Cekirge S, et al. Endovascular treatment of basilar and ICA termination aneurysms: effects of use of HydroCoils on treatment stability in a subgroup of patients prone to a higher recurrence rate. Neuroradiology 2007; 49:1015-21. Epub 2007 Oct 2

29. Gaba RC, Ansari SA, Roy SS, et al. Embolization of intracranial aneurysms with hydrogel-coated coils versus inert platinum coils: effects on packing density, coil length and quantity, procedural performance, cost, length of hospital stay and durability of therapy. Stroke 2006;37:1443-50

30. Deshaies EM, Adamo MA, Boulos AS. A prospective single-center analysis of the safety and efficacy of the HydroCoil embolic system for the treatment of intracranial aneurysms. J Neurosurg 2007;106:226-33

31. Fanning NF, Berentei Z, Brennan PR, et al. HydroCoil as an adjuvant to bare platinum coil treatment in 100 cerebral aneurysms. Neuroradiology 2007;49:139-48

32. Arthur AS, Wilson SA, Dixit S, et al. Hydrogel-coated coils for the treatment of cerebral aneurysms: preliminary results. Neurosurg Focus 2005;18:1-9

33. Berenstein A, Song JK, Niimi Y, et al. Treatment of cerebral aneurysms with hydrogel-coated platinum coils (HydroCoil): early single-center experience. AJNR Am J Neuroradiol 2006;27:1834-40

34. Gunnarsson T, Lurfan P, ter Brugg KG, et al. Treatment of intracranial aneurysms with hydrogel coated expandable coils. Can J Neurol Sci 2007;34:38-46

35. Ya P, Jing GX, Y Y, et al. Acute ruptured intracranial aneurysm packing with HydroCoil embolic system: initial clinical experience. The Neuroradiology Journal 2007;20:61-64
36. Kang HS, Han MH, Lee TH, et al. Embolization of intracranial aneurysms with hydrogel-coated coils: result of a Korean multicenter trial. Neurosurgery 2007;61:51-59

37. Cloft HJ, Kallmes DF. Aneurysm packing with HydroCoil embolic system versus platinum coils: Initial clinical experience. AJNR Am J Neuroradiol 2004;25:60-62

38. Katz JM, Tsiouris AJ, Biondi A. Advances in endovascular aneurysm treatment: are we making a difference? Neuroradiology 2005;47:695-701. Epub 2005 Jul 19

39. Liebig T, Henkes H, Fischer S, et al. Fibred electrolytically detachable platinum coils used for the endovascular treatment of intracranial aneurysms: initial experiences and mid-term results in $\mathbf{4 7 4}$ aneurysms. Intervent Neuroradiol 2004;10:5-26

40. Henkes H, Fischer S, Weber W, et al. Endovascular coil occlusion of 1811 intracranial aneurysms: early angiographic and clinical results. Neurosurgery 2004;54:268-80

41. Bendzsus M, Bartsch AJ, Solymosi L. Endovascular occlusion of aneurysms using a new bioactive coil: a matched pair analysis with bare platinum coils. Stroke 2007;28:55-57. Epub 2007 Aug 23

42. Butteriss D, Gholkar A, Mitra D, et al. Single-center experience of Cerecyte coils in the treatment of intracranial aneurysms: initial experience and early follow-up results. AJNR Am J Neuroradiol 2008;29:53-56. Epub 2007 Oct 5

43. Siddiqui AH, Koebbe C, Chen PR, et al. Prospective comparison of bare platinum and bioactive Micrus Cerecyte coil embolization for ruptured and unruptured intracranial aneurysms at the Jefferson Hospital for Neuroscience. Neurosurg 2006;59:813-14

44. Raymond J, Guilbert F, Weill A, et al. Long-term angiographic recurrences after selective endovascular treatment of aneurysms with detachable coils. Stroke 2003;34:1398-403

45. Raymond J, White P, Kallmes DF, et al. ICONE: an International Consortium of Neuro Endovascular Centres. Interventional Neuroradiology 2008;14:203-08

46. Clarke M. Systematic review of reviews of risk factors for intracranial aneurysms. Neuroradiology 2008;50:653-64

47. Brilstra EH, Rinkel GJE, van der GraafY, et al. Treatment of intracranial aneurysm by embolization with coils: a systematic review. Stroke 1999;30:470-76

48. van Rooij WJ, Sluzweski M, Bewte GN, et al. Procedural complications of coiling of ruptured intracranial aneurysms: incidence and risk factors in a consecutive series of 681 patients. AJNR Am J Neuroradiol 2006;27:1498-501

49. Vinuela F, Duckwiler G, Mawad M. Guglielmi detachable coil embolization of acute intracranial aneurysms: perioperative anatomical and clinical outcome in 403 patients. J Neurosurg 1997;86:475-82

50. Ng P, Khangure M, Phatouros CC. Endovascular treatment of intracrania aneurysm with Guglielmi detachable coils: analysis of midterm angiographic and clinical outcomes. Stroke 2002;33:210-17

51. Ross IB, Dhillon GS. Complications of endovascular treatment of cerebral aneurysms. Surg Neurol 2005;64:12-19

52. Cognard C, Weill A, Castaings L, et al. Intracranial berry aneurysms: angiographic and clinical results after endovascular treatment. Radiology 1998;206:449-510

53. Adams WM, Laitt RD. Aneurysm remnants. Neurointerventionist 2000;1:74-83

54. Murayama Y, Nien YL, Duckwiler G, et al. Guglielmi detachable coil embolization of cerebral aneurysms: 11 years' experience. J Neurosurg 2003;98:959-66

55. Campi A, Ramzi N, Molyneux AJ, et al. Retreatment of ruptured cerebral aneurysms in patients randomized by coiling or clipping in the International Subarachnoid Aneurysm Trial (ISAT). Stroke 2007;38:1538-44. Epub 2007 Mar 29

56. Johnston SC, Dowd CF, Higashida RT, et al. Predictors of rehemorrhage after treatment of ruptured intracranial aneurysms: The Cerebral Aneurysms Rerupture After Treatment (CARAT) Study. Stroke 2008;39:120-25. Epub 2007 Nov 29

57. Cloft HJ. What is all the hype about? AJNR Am J Neuroradiol 2008;29:1604 